\title{
CONTINUOUS WRIST BLOOD PRESSURE MEASUREMENT WITH ULTRASOUND
}

\author{
Weber $\mathrm{S}^{1}$, Scharfschwerdt $\mathrm{P}^{1}$, Seel $\mathrm{T}^{2}$, Schauer $\mathrm{T}^{2}$, Kertzscher $\mathrm{U}^{1}$, Affeld $\mathrm{K}^{1}$ \\ ${ }^{1}$ Biofluid Mechanics Lab, Charité Universitätsmedizin Berlin, Germany \\ ${ }^{2}$ Control Systems Group, Technische Universität Berlin, Germany \\ sarah.weber@charite.de
}

\begin{abstract}
Blood pressure is an important parameter for the development of cardiovascular disease. By monitoring the blood pressure over 24 hours hypertension can be detected and treatment can be started. A new noninvasive long term blood pressure measurement method measures the blood pressure continuously on the wrist using ultrasound, a small balloon and a controller. The pressure is controlled with a voice coil actuator. The wrist blood pressure changes during motion. These movements are assessed with a water filled tube to calibrate the measurement to aortic blood pressure.
\end{abstract}

Keywords: blood pressure, ultrasound, motion artefacts

\section{Introduction}

Blood pressure is an important parameter for the development of cardiovascular disease. A long term measurement provides a more detailed and representative result than a single measurement with the RIVA-ROCCI method using an arm cuff [1]. Furthermore, ambulatory $24 \mathrm{~h}$ blood pressure measurement with an arm cuff is stressful for the patient and disturbs his activities.

A new method for blood pressure measurement uses the radial artery at the wrist. The measurement principle is based on the RIVA-ROCCI and the PEŇÁZ principle [3]. In the RIVA-RocCI method a cuff is used to compress the upper arm arteries and measure the blood pressure by detecting the pulsation of blood through the vessel. In contrast to the traditional measurement, a small pressure balloon occludes the radial artery on the wrist. The radial artery is a small, superficial artery where only a small volume is needed to occlude the blood flow making the method more comfortable for the patient.

In the PEŃÁZ principle the pressure in a finger cuff is continuously adjusted to maintain a constant vascular volume. This can lead to ischemia. In our technique, the pressure in the balloon is controlled to generate a constant blood velocity in the vessel. The blood velocity is detected by ultrasound. A control loop is used to evaluate the ultrasound signal of radial blood flow to continuously measure the radial blood pressure.

\section{Methods}

A drawing of the device is shown in Figure 1. It consists of a polyurethane balloon with a size of $40 \times 20 \mathrm{~mm}^{2}$ which is placed over the radial artery on the wrist. The balloon is inflated by a voice coil actuator which is controlled by a microcontroller.

An ultrasound (US) sensor is attached to the radial artery downstream of the balloon measuring the blood velocity with continuous wave DOPPLER ultrasound. A rectangular piezo ceramic of $10 \times 3 \mathrm{~mm}^{2}$ size is used to generate a larger beam volume. The ceramic is divided into three parts for individual activation. This optimizes the form of the beam in accordance to anatomical conditions. This increases the signal-noise-ratio which is a crucial parameter for the device. The ultrasound sensors were fabricated by Fraunhofer Institut für Biomedizinische Technik, St. Ingbert, Germany.

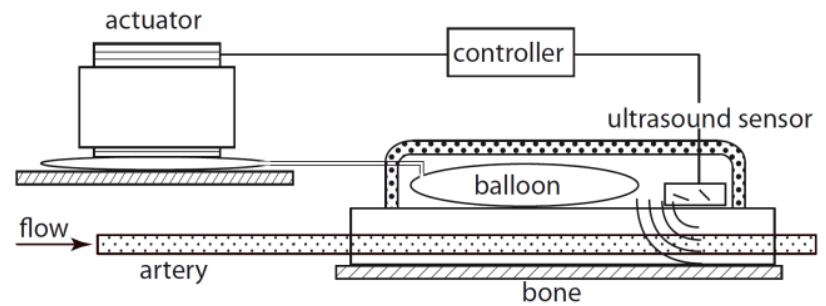

Figure 1: Drawing of the device. The ultrasound sensor detects the blood velocity in the radial artery; the controller activates the voice coil actuator to inflate the balloon to reduce but not stop the blood flow in the artery.

A controller is used to continuously adjust the detected ultrasound signal by controlling the balloon pressure. An iterative learning controller (ILC) and a proportionalintegral-derivative controller were implemented on the microcontroller Arduino DUE R3 board, Arduino ${ }^{\mathrm{TM}}$, Italy. The controller setup was tested on the experimental setup described in [2].

The principle of the technique is described in [2]. The balloon pressure is controlled to generate a constant blood velocity close to but always above zero in the vessel. This is required to guarantee the detection of an US signal at all times. The balloon pressure therefore follows the arterial blood pressure - a continuous measurement of radial blood pressure is found.

The measurement site is located on the wrist but aortic blood pressure should be assessed. The radial blood pressure is calibrated to aortic pressure by measuring the pressure changes induced by motion with a water filled tube. The tube runs parallel to the arteries in the arm. The pressure in the tube is changed by height differences and acceleration which also change the blood pressure between heart and wrist, [4]. A schematic drawing of the tube is shown in Figure 4.

\section{Results}

The device is shown in Figure 2 and has a total weight of $1,3 \mathrm{~kg}$. The wrist band including the ultrasound sensor and balloon weighs $30 \mathrm{~g}$. The voice coil actuator (340 g) and US-electronics $(50 \mathrm{~g})$ will be attached to the lower arm. The microcontroller (60 g), voice coil power amplifier 
( $320 \mathrm{~g})$ and lithium-ion battery $(520 \mathrm{~g})$ will be carried in a bag attached to the hip.

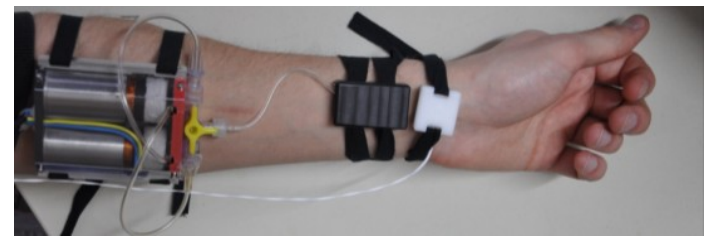

Figure 2: Wrist band and voice coil actuator of the device. The ultrasound sensor and balloon are attached over the radial artery and held in position by the wrist band. The voice coil actuator is attached to the lower arm. The US-electronics (not shown) are located on the other side of the wrist.

The results of the iterative learning controller in the experimental setup are shown in Figure 3. The graphs show the difference between a feedback controller (indicated with a low level graph in Figure 3) and the ILC (identified in Figure 3). The shape of the voice coil pressure and model arterial pressure look similar during the experiment but only during the ILC phase, the flow signal is adjusted to the desired threshold value, [5]. During the feedback control, the voice coil pressure is delayed to the arterial pressure. Therefore, the flow is not sufficiently decreased.
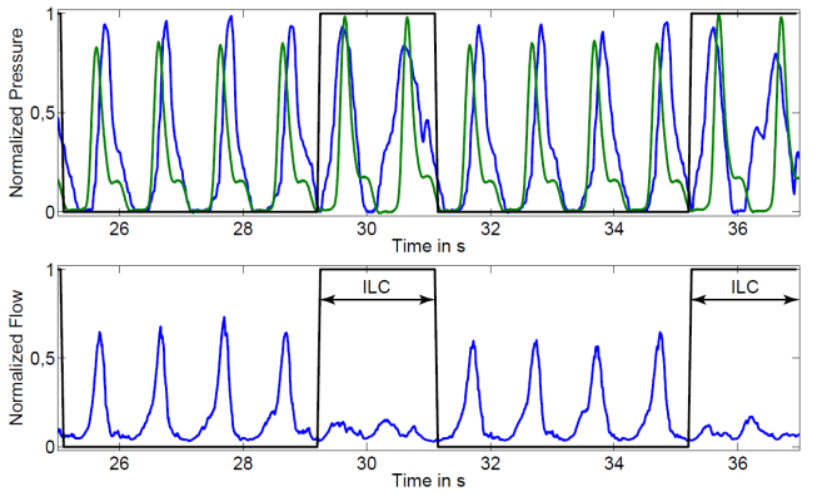

Figure 3: Top: Normalized voice coil pressure (blue) and model arterial pressure (green). Bottom: Normalized flow signal on experimental setup. Feedback controller is indicated with the black low level graph, ILC is indicated with the black high level graph.

Lifting the arm leads to blood pressure changes in the radial artery. Figure 4, right shows the pressure differences in the calibration water tube that also affect the arterial pressure.
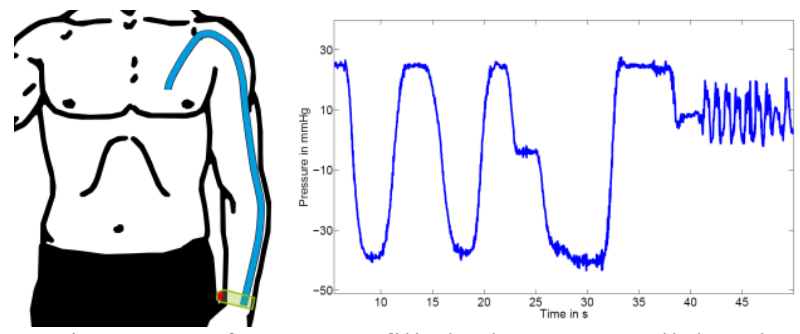

Figure 4: Left: A water filled tube runs parallel to the arteries from the heart to the wrist. Right: Pressure in the water tube on a subject repeatedly lifting the arm.

\section{Discussion}

The attachment of the balloon to the wrist is crucial for the measurement technique. The balloon has to be pressed to the skin at all times also during movement. The wrist band only fastened by fabric straps is not powerful enough to ensure the balloon pressure to occlude the artery.

The results of the iterative learning controller are promising, however, further analysis is needed.

There are two possibilities to place the voice coil actuator: in the bag on the hip or at the lower arm. In the case that the actuator is situated in a bag, the supply tube must be inelastic to guarantee a fast transfer of pressure to the balloon.

If the actuator is situated on the lower arm, the additional weight leads to fewer motion artefacts.

The power amplifier for the voice coil actuator needs to be reduced in size and weight. An appropriate battery has to be adjusted for the device.

\section{Acknowledgement}

The project was funded by the German federal ministry of education and research (BMBF) under the promotional reference 01 EZ 0918.

\section{Bibliography}

[1] Y. Imai: Clinical significance and cost-effectiveness of 24-hour ambulatory blood pressure monitoring. Tohoku J. Exp. Med., vol. 176, pp. 1-15, 1995

[2] S. Weber et. al.: Continuous blood pressure measurement with ultrasound. Biomed Tech (Berl), 57 Suppl 1, 2012

[3] J. Peňáz: Photoelectric Measurement of blood pressure, volume and flow in the finger. Digest of the 10th international conference on medical and biological engineering, Dresden, 1973

[4] S. Weber et. al.: Measurement of motion artefacts in noninvasive blood pressure measurement. Biosignalverarbeitung und Magnetische Methoden in der Medizin, 2012

[5] T. Seel et. al.: Iterative learning cascade control of continuous noninvasive blood pressure measurement. IEEE SMC 2013, submitted 